\title{
Multiplication operators on weighted Bloch spaces of the first Cartan domains
}

Zhi-jie Jiang ${ }^{1 *}$

\author{
Correspondence: matjzj@126.com \\ 1 School of Mathematics and \\ Statistics, Sichuan University of \\ Science and Engineering, Zigong, \\ Sichuan, 643000, P.R. China
}

\begin{abstract}
Let $\Re(m, n)$ be the first Cartan domain. Motivated by some results of the multiplication operators on the holomorphic function spaces on the unit ball of $\mathbb{C}^{n}$, we study multiplication operators on weighted Bloch spaces of the first Cartan domain and obtain some necessary or sufficient conditions for the boundedness and compactness in this paper.
\end{abstract}

MSC: Primary 42A18; secondary 47B33

Keywords: Multiplication operator; First Cartan domain; Weighted Bloch space; Boundedness; Compactness

\section{Introduction}

The well-known first Cartan domain (see [22]) is defined as

$$
\Re_{I}(m, n)=\left\{Z=\left(z_{i j}\right)_{m \times n} \in \mathbb{C}^{m \times n}: I-Z \bar{Z}^{T}>0\right\},
$$

where $\bar{Z}$ denotes the conjugate of the matrix $Z, Z^{T}$ denotes the transpose of $Z$, and $m, n$ are positive integers. For the sake of convenience, it is denoted by $\Re_{I}$.

Let $H\left(\Re_{I}\right)$ be the space of all holomorphic functions on $\Re_{I}$. For $\alpha \geq 0$, the weighted-type space $H_{\alpha}^{\infty}\left(\Re_{I}\right)$ on $\Re_{I}$ consists of all $f \in H\left(\Re_{I}\right)$ such that

$$
\|f\|_{H_{\alpha}^{\infty}\left(\Re_{I}\right)}=\sup _{Z \in \Re_{I}}\left[\operatorname{det}\left(I-Z \bar{Z}^{T}\right)\right]^{\alpha}|f(Z)|<+\infty .
$$

The little weighted-type space $H_{\alpha, 0}^{\infty}\left(\Re_{I}\right)$ on $\Re_{I}$ consists of all $f \in H\left(\Re_{I}\right)$ such that

$$
\lim _{Z \rightarrow \partial \Re_{I}}\left[\operatorname{det}\left(I-Z \bar{Z}^{T}\right)\right]^{\alpha}|f(Z)|=0
$$

If $\alpha=0$, then $H_{\alpha}^{\infty}\left(\Re_{I}\right)$ is denoted as $H^{\infty}\left(\Re_{I}\right)$ and $H_{\alpha, 0}^{\infty}\left(\Re_{I}\right)$ is denoted as $H_{0}^{\infty}\left(\Re_{I}\right)$. The weighted-type spaces on the unit disk or the unit ball frequently appear; see, for example, $[6,11,12,16,17,19]$.

Let $\mathbb{B}=\left\{z \in \mathbb{C}^{n}:|z|<1\right\}$ be the open unit ball of $\mathbb{C}^{n}$. It is obvious that $\mathbb{B}=\Re_{I}(1, n)$, which shows that $\Re_{I}(m, n)$ is a generalization of $\mathbb{B}$. The weighted Bloch space on $\mathbb{B}$, usually

(c) The Author(s) 2020. This article is licensed under a Creative Commons Attribution 4.0 International License, which permits use, sharing, adaptation, distribution and reproduction in any medium or format, as long as you give appropriate credit to the original author(s) and the source, provide a link to the Creative Commons licence, and indicate if changes were made. The images or other third party material in this article are included in the article's Creative Commons licence, unless indicated otherwise in a credit line to the material. If material is not included in the article's Creative Commons licence and your intended use is not permitted by statutory regulation or exceeds the permitted use, you will need to obtain permission directly from the copyright holder. To view a copy of this licence, visit http://creativecommons.org/licenses/by/4.0/. 
denoted by $\mathcal{B}^{\alpha}(\mathbb{B})$, consists of all $f \in H(\mathbb{B})$ such that

$$
b(f):=\sup _{z \in \mathbb{B}}\left(1-|z|^{2}\right)^{\alpha}|\nabla f(z)|<+\infty,
$$

where

$$
\nabla f(z)=\left(\frac{\partial f(z)}{\partial z_{1}}, \frac{\partial f(z)}{\partial z_{2}}, \ldots, \frac{\partial f(z)}{\partial z_{n}}\right) .
$$

It is well known that the quantity $b(f)$ is a seminorm of $\mathcal{B}^{\alpha}(\mathbb{B})$ and under the norm

$$
\|f\|_{\mathcal{B}^{\alpha}(\mathbb{B})}=|f(0)|+b(f),
$$

$\mathcal{B}^{\alpha}(\mathbb{B})$ is a Banach space. The weighted Bloch spaces also frequently appear in the literature; see, for example, [2, 13-15, 27].

Similarly, the weighted Bloch space $\mathcal{B}^{\alpha}\left(\Re_{I}\right)$ on $\Re_{I}$ consists of all $f \in H\left(\Re_{I}\right)$ such that

$$
s(f):=\sup _{z \in \Re_{I}}\left[\operatorname{det}\left(I-Z \bar{Z}^{T}\right)\right]^{\alpha}|\nabla f(Z)|<+\infty .
$$

Under the norm

$$
\|f\|_{\mathcal{B}^{\alpha}\left(\Re_{I}\right)}=|f(0)|+s(f),
$$

$\mathcal{B}^{\alpha}\left(\Re_{I}\right)$ is a Banach space. The little Bloch space $\mathcal{B}_{0}^{\alpha}\left(\Re_{I}\right)$ on $\Re_{I}$ consists of all $f \in H\left(\Re_{I}\right)$ such that

$$
\lim _{Z \rightarrow \partial \Re_{I}}\left[\operatorname{det}\left(I-Z \bar{Z}^{T}\right)\right]^{\alpha}|\nabla f(Z)|=0 .
$$

Let $X$ and $Y$ be two function spaces on $\mathfrak{\Re}_{I}$. If it follows that $\psi f \in Y$ for all $f \in X$, then $\psi$ is called a multiplier from $X$ to $Y$, and usually $M_{\psi}: f \mapsto \psi f$ is called a multiplication operator from $X$ to $Y$. In general, $\psi f$ cannot necessarily belong to $Y$ for some $f \in X$. In order to explain this fact, we need to introduce the Bloch space $\mathcal{B}\left(U^{2}\right)$ (see [24]), where $U^{2}=\left\{z=\left(z_{1}, z_{2}\right):\left|z_{1}\right|<1,\left|z_{2}\right|<1\right\}$ is the unit polydisk in $\mathbb{C}^{2}$. We say a holomorphic function $f$ belongs to $\mathcal{B}\left(U^{2}\right)$ if $f$ satisfies the condition

$$
\sup _{z \in U^{2}}\left[\left(1-\left|z_{1}\right|^{2}\right)\left|\frac{\partial f}{\partial z_{1}}(z)\right|+\left(1-\left|z_{2}\right|^{2}\right)\left|\frac{\partial f}{\partial z_{2}}(z)\right|\right]<+\infty .
$$

If we consider

$$
f\left(z_{1}, z_{2}\right)=\log \frac{1}{1-z_{1}}+\log \frac{1}{1-z_{2}}
$$

in $\mathcal{B}\left(U^{2}\right)$, it is easy to see that $\psi f$ does not belong to $\mathcal{B}\left(U^{2}\right)$ for $\psi(z)=z_{1}$. Hence, a natural problem is to find some conditions when $\psi$ satisfies $M_{\psi} f \in Y$ for all $f \in X$. It is well known that the theory of the multipliers or multiplication operators on function spaces has been studied for a long time. In 1966, Talyor started an investigation of the multipliers on $D_{\alpha}$ in 
[21]. Later on, for example, Stegenga considered the multipliers of the Dirichlet space in [10]. Now multipliers or multiplication operators between or on various function spaces of the classical domains have been studied by many authors (see, for example, $[5,7,23,26]$ ). But beyond that, there is a great interest in some generalizations of the multiplication operators on classical domains (for example, see $[1,4,8,15,18]$ for the weighted composition operators). However, we do not find any result of multipliers or multiplication operators on the holomorphic function spaces of the first Cartan domain. In this paper, we study the multiplication operators on weighted Bloch spaces of the first Cartan domain and obtain some necessary or sufficient conditions for the boundedness and compactness.

For $Z=\left(z_{i j}\right)_{m \times n} \in \mathbb{C}^{m \times n}$, let $|Z|^{2}=\sum_{\substack{1 \leq i \leq m \\ 1 \leq j \leq n}}\left|z_{i j}\right|^{2}$. Constants are denoted by $C$, they are positive and may differ from one occurrence to the next. The notation $a \lesssim b$ means that there exists a positive constant $C$ independent of the essential variables in the quantities $a$ and $b$ such that $a \leq C b$.

\section{Some lemmas}

First, we have the following obvious result.

Lemma 1 Let $Z=\left(z_{i j}\right)_{m \times n} \in \Re_{I}$, then $\left|z_{i j}\right|<1$ for all $i$ and $j$.

Proof Let $Z=\left(z_{i j}\right)_{m \times n} \in \Re_{I}$. Then $I-Z \bar{Z}^{T}>0$. So, we have $1-\sum_{j=1}^{n}\left|z_{i j}\right|^{2}>0$ for $i=$ $1,2, \ldots, n$, from which the desired result follows.

By a direct calculation, we obtain the following formula.

Lemma 2 Let $f \in H\left(\Re_{I}\right)$. Then, for all $Z \in \Re_{I}$, it follows that

$$
\nabla\left(M_{\psi} f\right)(Z)=f(Z) \nabla \psi(Z)+\nabla f(Z) \psi(Z)
$$

We need the following result (see [20]) to obtain the point evaluation estimate for the Bloch functions.

Lemma 3 Let $Z \in \Re_{I}$. Then there exist two unitary matrices $U$ and $V$ such that

$$
Z=U\left(\begin{array}{ccccccc}
\lambda_{1} & 0 & \cdots & 0 & 0 & \cdots & 0 \\
0 & \lambda_{2} & \cdots & 0 & 0 & \cdots & 0 \\
\vdots & \vdots & \ddots & \vdots & \vdots & \cdots & 0 \\
0 & 0 & \cdots & \lambda_{m} & 0 & \cdots & 0
\end{array}\right) V
$$

where $1>\lambda_{1} \geq \lambda_{2} \geq \cdots \geq \lambda_{m} \geq 0$ and $\lambda_{1}^{2}, \ldots, \lambda_{m}^{2}$ are eigenvalues of $Z \bar{Z}^{T}$.

Lemma 4 Let $\alpha>0$. Then there exists a positive constant $C$ independent of $f \in \mathcal{B}^{\alpha}\left(\Re_{I}\right)$ and $Z \in \Re_{I}$ such that

$$
|f(Z)| \leq \begin{cases}C\|f\|_{\mathcal{B}^{\alpha}\left(\Re_{I}\right)}, & 0<m \alpha<1, \\ C\|f\|_{\mathcal{B}^{\alpha}\left(\Re_{I}\right)} \log \frac{2}{\operatorname{det}\left(I-Z \bar{Z}^{T}\right)}, & m \alpha=1, \\ C\|f\|_{\mathcal{B}^{\alpha}\left(\Re_{I}\right)} \frac{1}{\left[\operatorname{det}\left(I-Z \bar{Z}^{T}\right)\right]^{m \alpha-1}}, & m \alpha>1 .\end{cases}
$$


Proof If $Z=0$, then the result holds obviously. Now, assume that $Z=\left(z_{i j}\right)_{m \times n} \neq 0$. It follows from Lemma 3 that there exist two unitary matrices $U$ and $V$ such that

$$
Z=U\left(\begin{array}{ccccccc}
\lambda_{1} & 0 & \cdots & 0 & 0 & \cdots & 0 \\
0 & \lambda_{2} & \cdots & 0 & 0 & \cdots & 0 \\
\vdots & \vdots & \ddots & \vdots & \vdots & \cdots & 0 \\
0 & 0 & \cdots & \lambda_{m} & 0 & \cdots & 0
\end{array}\right) V
$$

where $1>\lambda_{1} \geq \lambda_{2} \geq \cdots \geq \lambda_{m} \geq 0$ and $\lambda_{1}^{2}, \ldots, \lambda_{m}^{2}$ are eigenvalues of $Z \bar{Z}^{T}$. By (2), we have

$$
1-t^{2} Z \bar{Z}^{T}=U\left(\begin{array}{cccc}
1-t^{2} \lambda_{1}^{2} & 0 & \cdots & 0 \\
0 & 1-t^{2} \lambda_{2}^{2} & \cdots & 0 \\
\vdots & \vdots & \ddots & \vdots \\
0 & 0 & \cdots & 1-t^{2} \lambda_{m}^{2}
\end{array}\right) \bar{U}^{T}
$$

It follows from (3) that

$$
\left[\operatorname{det}\left(I-t^{2} Z \bar{Z}^{T}\right)\right]^{\alpha}=\prod_{i=1}^{m}\left(1-t^{2} \lambda_{i}^{2}\right)^{\alpha}
$$

Assume $t \in[0,1]$. Since $\lambda_{1} \geq \lambda_{2} \geq \cdots \geq \lambda_{m} \geq 0$, for each $i \in\{1,2, \ldots, m\}$, we have

$$
1-t^{2} \lambda_{i}^{2}=\left(1-t \lambda_{i}\right)\left(1+t \lambda_{i}\right) \geq\left(1-t \lambda_{i}\right)
$$

By this, we have

$$
\left[\operatorname{det}\left(I-t^{2} Z \bar{Z}^{T}\right)\right]^{\alpha}=\prod_{i=1}^{m}\left(1-t^{2} \lambda_{i}^{2}\right)^{\alpha} \geq\left(1-t \lambda_{i}\right)^{m \alpha}
$$

From the facts

$$
Z \bar{Z}^{T}=U\left(\begin{array}{cccc}
\lambda_{1}^{2} & 0 & \cdots & 0 \\
0 & \lambda_{2}^{2} & \cdots & 0 \\
\vdots & \vdots & \ddots & \vdots \\
0 & 0 & \cdots & \lambda_{m}^{2}
\end{array}\right) \bar{U}^{T}
$$

and $|Z|^{2}=\operatorname{tr}\left(Z \bar{Z}^{T}\right)$, we obtain

$$
|Z|^{2}=\sum_{i=1}^{m} \lambda_{i}^{2} \leq m \lambda_{1}^{2}
$$

Then, from (4) and (5) we obtain

$$
|f(Z)|=\left|f(0)+\int_{0}^{1}\langle\nabla f(t Z), \bar{Z}\rangle \mathrm{d} t\right| \leq|f(0)|+\int_{0}^{1}|\nabla f(t Z)||\bar{Z}| \mathrm{d} t
$$




$$
\begin{aligned}
& \leq|f(0)|+\int_{0}^{1} \frac{|Z| \mathrm{d} t}{\left[\operatorname{det}\left(I-t^{2} Z \bar{Z}^{T}\right)\right]^{\alpha}}\|f\|_{\mathcal{B}^{\alpha}\left(\Re_{I}\right)} \\
& =|f(0)|+\int_{0}^{1} \frac{|Z| \mathrm{d} t}{\prod_{j=1}^{m}\left(1-t^{2} \lambda_{j}^{2}\right)^{\alpha}}\|f\|_{\mathcal{B}^{\alpha}\left(\Re_{I}\right)} \\
& \leq\left[1+\int_{0}^{1} \frac{\sqrt{m} \lambda_{1}}{\left(1-\lambda_{1} t\right)^{m \alpha}} \mathrm{d} t\right]\|f\|_{\mathcal{B}^{\alpha}\left(\Re_{I}\right)} \\
& =\left[1+\int_{0}^{\lambda_{1}} \frac{\sqrt{m}}{(1-t)^{m \alpha}} \mathrm{d} t\right]\|f\|_{\mathcal{B}^{\alpha}\left(\Re_{I}\right)} \\
& \leq \begin{cases}C\|f\|_{\mathcal{B}^{\alpha}\left(\Re_{I}\right)}, & 0<m \alpha<1, \\
C\|f\|_{\mathcal{B}^{\alpha}\left(\Re_{I}\right)} \log \frac{1}{1-\lambda_{1}}, & m \alpha=1, \\
C\|f\|_{\mathcal{B}^{\alpha}\left(\Re_{I}\right)} \frac{1}{\left(1-\lambda_{1}\right)^{m \alpha-1}}, & m \alpha>1\end{cases} \\
& \leq \begin{cases}C\|f\|_{\mathcal{B}^{\alpha}\left(\Re_{I}\right)}, & 0<m \alpha<1, \\
C\|f\|_{\mathcal{B}^{\alpha}\left(\Re_{I}\right)} \log \frac{2}{\operatorname{det}\left(I-Z \bar{Z}^{T}\right)}, & m \alpha=1, \\
C\|f\|_{\mathcal{B}^{\alpha}\left(\Re_{I}\right)} \frac{1}{\left[\operatorname{det}\left(I-Z \bar{Z}^{T}\right)\right]^{m \alpha-1}}, & m \alpha>1,\end{cases}
\end{aligned}
$$

where (7) is obtained by (6) by using the elementary fact

$$
1-\lambda_{1} \geq \frac{1-\lambda_{1}^{2}}{2} \geq \frac{1}{2} \operatorname{det}\left(I-Z \bar{Z}^{T}\right) .
$$

This completes the proof.

Remark 1 In Lemma 4, there exists a parameter $m$ which maybe is the biggest difference from the weighted Bloch spaces on the unit ball. Unfortunately, we do not find an effective method to avoid it. However, this also shows that this result is a generalization of the corresponding result on $\mathcal{B}^{\alpha}(\mathbb{B})$.

In order to study the compactness of the operator $M_{\psi}$ on $\mathcal{B}^{\alpha}\left(\Re_{I}\right)$, we need the following result which is similar to Proposition 3.11 in [3].

Lemma 5 Let $\alpha>0$ and $\psi$ be a holomorphic function on $\mathfrak{R}_{I}$. Then the bounded operator $M_{\psi}$ is compact on $\mathcal{B}^{\alpha}\left(\Re_{I}\right)$ if and only iffor every bounded sequence $\left\{f_{n}\right\}$ in $\mathcal{B}^{\alpha}\left(\Re_{I}\right)$ such that $f_{n} \rightarrow 0$ uniformly on any compact subset of $\Re_{I}$ as $n \rightarrow \infty$, it follows that

$$
\lim _{n \rightarrow \infty}\left\|M_{\psi} f_{n}\right\|_{\mathcal{B}^{\alpha}\left(\Re_{I}\right)}=0
$$

Proof Suppose that the bounded operator $M_{\psi}$ is compact on $\mathcal{B}^{\alpha}\left(\Re_{I}\right)$. Let $\left\{f_{n}\right\}$ be a bounded sequence in $\mathcal{B}^{\alpha}\left(\Re_{I}\right)$ such that $f_{n} \rightarrow 0$ uniformly on any compact subset of $\Re_{I}$ as $n \rightarrow \infty$. If $\left\|M_{\psi} f_{n}\right\|_{\mathcal{B}^{\alpha}\left(\Re_{I}\right)} \nrightarrow 0$ as $n \rightarrow \infty$, then there exists a subsequence $\left\{f_{n_{j}}\right\}$ of $\left\{f_{n}\right\}$ such that

$$
\inf _{n \in N}\left\|M_{\psi} f_{n_{j}}\right\|_{\mathcal{B}^{\alpha}\left(\Re_{I}\right)}>0 .
$$


Since $M_{\psi}$ is compact on $\mathcal{B}^{\alpha}\left(\Re_{I}\right)$, there exist a function $g \in \mathcal{B}^{\alpha}\left(\Re_{I}\right)$ and a subsequence of $\left\{f_{n_{j}}\right\}$ (without loss of generality, still written by $\left\{f_{n_{j}}\right\}$ ) such that

$$
\lim _{j \rightarrow \infty}\left\|M_{\psi} f_{n_{j}}-g\right\|_{\mathcal{B}^{\alpha}\left(\Re_{I}\right)}=0 .
$$

Let $K$ be a compact subset of $\Re_{I}$. From Lemma 4, it follows that $M_{\psi} f_{n_{j}}-g \rightarrow 0$ uniformly on $K$ as $j \rightarrow \infty$. From this, for $\varepsilon>0$, there exists a positive integer $N_{1}$ such that

$$
\left|\psi(Z) f_{k_{j}}(Z)-g(Z)\right|<\varepsilon
$$

for all $Z \in K$, whenever $j>N_{1}$. Since $f_{n_{j}} \rightarrow 0$ uniformly on $K$ as $j \rightarrow \infty$, also there exists a positive integer $N_{2}$ such that $\left|f_{n_{j}}(Z)\right|<\varepsilon$ for all $Z \in K$, whenever $j>N_{2}$. Let $N=\max \left\{N_{1}, N_{2}\right\}$ and $M=\max _{Z \in K}|\psi(Z)|$. From (9), we have

$$
|g(Z)| \leq M\left|f_{n_{j}}(Z)\right|+\varepsilon<(M+1) \varepsilon
$$

for all $Z \in K$, whenever $j>N$. From (10) and the arbitrariness of $\varepsilon$, we obtain $g(Z)=0$ for all $Z \in K$, which leads to $g \equiv 0$ on $\Re_{I}$. This shows that $\lim _{j \rightarrow \infty}\left\|M_{\psi} f_{n_{j}}\right\|_{\mathcal{B}^{\alpha}\left(\Re_{I}\right)}=0$ which contradicts (8).

Now suppose that $\left\{f_{n}\right\}$ is a bounded sequence in $\mathcal{B}^{\alpha}\left(\Re_{I}\right)$. Then it is locally uniformly bounded on $\Re_{I}$, which shows that there exists a subsequence $\left\{f_{n_{j}}\right\}$ of $\left\{f_{n}\right\}$ such that $f_{n_{j}} \rightarrow$ $f$ uniformly on every compact subset of $\Re_{I}$ as $j \rightarrow \infty$. From this, we have $f_{n_{j}}-f \rightarrow 0$ uniformly on every compact subset of $\Re_{I}$ as $j \rightarrow \infty$. Consequently, we obtain

$$
\lim _{j \rightarrow \infty}\left\|M_{\psi}\left(f_{n_{j}}-f\right)\right\|_{\mathcal{B}^{\alpha}\left(\Re_{I}\right)}=\lim _{j \rightarrow \infty}\left\|M_{\psi} f_{n_{j}}-M_{\psi} f\right\|_{\mathcal{B}^{\alpha}\left(\Re_{I}\right)}=0,
$$

which shows that $M_{\psi}$ is compact on $\mathcal{B}^{\alpha}\left(\Re_{I}\right)$.

In the studies of the several complex variables, the mathematician Loo-Keng Hua found the following matrix inequality in 1955.

Lemma 6 Let $I-A \bar{A}^{T}$ and $I-B \bar{B}^{T}$ be two Hermitian and positive definite matrices. Then

$$
\operatorname{det}\left(I-A \bar{A}^{T}\right) \operatorname{det}\left(I-B \bar{B}^{T}\right) \leq\left|\operatorname{det}\left(I-A \bar{B}^{T}\right)\right|^{2}
$$

By the way, as an easy application of Lemma 6, we see that, for each $Z, S \in \mathfrak{R}_{I}$, the matrix $I-Z \bar{S}^{T}$ is reversible. We also have the following result (see [20]).

Lemma 7 There exists a positive constant $C$ independent of all $Z, S \in \Re_{I}$ such that

$$
\left|\operatorname{det}\left(I-Z \bar{S}^{T}\right)\right|\left\{\sum_{\substack{1 \leq i \leq m \\ 1 \leq j \leq n}}\left|\operatorname{tr}\left[\left(I-Z \bar{S}^{T}\right)^{-1} I_{i j} \bar{S}^{T}\right]\right|^{2}\right\}^{\frac{1}{2}} \leq C,
$$

where $I_{i j}$ is an $m \times n$ matrix whose element of the ith row and the jth column is 1 , and the other elements are 0 . 
Let $S$ be a fixed point in $\Re_{I}$. If $\alpha=\frac{1}{2}$, on $\Re_{I}$ we define the function

$$
f_{S}(Z)=\left[\operatorname{det}\left(I-S \bar{S}^{T}\right)\right]^{\frac{1}{2}} \log \frac{2}{\operatorname{det}\left(I-Z \bar{S}^{T}\right)},
$$

and if $\alpha \neq \frac{1}{2}$, on $\Re_{I}$ we define the function

$$
g_{S}(Z)=\frac{1}{1-2 \alpha} \frac{\left[\operatorname{det}\left(I-S \bar{S}^{T}\right)\right]^{\alpha}}{\left[\operatorname{det}\left(I-Z \bar{S}^{T}\right)\right]^{2 \alpha-1}} .
$$

To prove that $f_{S}$ belongs to $\mathcal{B}^{\frac{1}{2}}\left(\Re_{I}\right)$ (or $g_{S}$ belongs to $\mathcal{B}^{a}\left(\Re_{I}\right)$ for $\alpha \neq \frac{1}{2}$ ), let us recall the definition of the function matrix derivative.

Let

$$
Y(x)=\left(\begin{array}{cccc}
y_{11}(x) & y_{12}(x) & \cdots & y_{1 n}(x) \\
y_{21}(x) & y_{22}(x) & \cdots & y_{2 n}(x) \\
\vdots & \vdots & \vdots & \vdots \\
y_{n 1}(x) & y_{n 2}(x) & \cdots & y_{n n}(x)
\end{array}\right)
$$

and each $y_{i j}(x)$ be differentiable on the interval $I$. Then the well-known derivative of $Y(x)$ is defined as

$$
\frac{d Y(x)}{d x}=\left(\begin{array}{cccc}
y_{11}^{\prime}(x) & y_{12}^{\prime}(x) & \cdots & y_{1 n}^{\prime}(x) \\
y_{21}^{\prime}(x) & y_{22}^{\prime}(x) & \cdots & y_{2 n}^{\prime}(x) \\
\vdots & \vdots & \vdots & \vdots \\
y_{n 1}^{\prime}(x) & y_{n 2}^{\prime}(x) & \cdots & y_{n n}^{\prime}(x)
\end{array}\right) .
$$

If we regard $\operatorname{det}\left(I-Z \bar{S}^{T}\right)$ as a function of $Z$, we have the following result.

Lemma 8 Let $S$ be a fixed point in $\Re_{I}$. Then on $\Re_{I}$ it follows that

$$
\frac{\partial \operatorname{det}\left(I-Z \bar{S}^{T}\right)}{\partial z_{i j}}=\operatorname{det}\left(I-Z \bar{S}^{T}\right) \operatorname{tr}\left[\left(I-Z \bar{S}^{T}\right)^{-1} I_{i j} \bar{S}^{T}\right] .
$$

Proof Let $A(x)$ be a differentiable function matrix and $\operatorname{det} A(x) \neq 0$ for each $x \in I$. Then by the formula (see [9])

$$
\frac{d \operatorname{det} A(x)}{d x}=\operatorname{det} A(x) \operatorname{tr}\left[A(x)^{-1} \frac{d A(x)}{d x}\right]
$$

we have

$$
\frac{\partial \operatorname{det}\left(I-Z \bar{S}^{T}\right)}{\partial z_{i j}}=\operatorname{det}\left(I-Z \bar{S}^{T}\right) \operatorname{tr}\left[\left(I-Z \bar{S}^{T}\right)^{-1} \frac{\partial\left(I-Z \bar{S}^{T}\right)}{\partial z_{i j}}\right] .
$$

By the definition of the function matrix derivative, it is easy to see that

$$
\frac{\partial\left(I-Z \bar{S}^{T}\right)}{\partial z_{i j}}=I_{i j} \bar{S}
$$

From (13) and (14), the desired result follows. 
Lemma 9 Let $\alpha>0$. For the fixed point $S \in \mathfrak{R}_{I}$, the following statements hold.

(i) If $\alpha=\frac{1}{2}$, then the function $f_{S}$ belongs to $\mathcal{B}^{\frac{1}{2}}\left(\Re_{I}\right)$. Moreover,

$$
\sup _{S \in \Re_{I}^{i}}\left\|f_{S}\right\|_{\mathcal{B}^{\frac{1}{2}\left(\Re_{I}\right)}} \lesssim 1
$$

(ii) If $\alpha \neq \frac{1}{2}$, then the function $g_{S}$ belongs to $\mathcal{B}^{\alpha}\left(\Re_{I}\right)$. Moreover,

$$
\sup _{S \in \Re_{I}^{2}}\left\|g_{S}\right\|_{\mathcal{B}^{\alpha}\left(\Re_{I}\right)} \lesssim 1
$$

Proof We first prove (i). By Lemma 8, we have

$$
\frac{\partial f_{S}(Z)}{\partial z_{i j}}=\left[\operatorname{det}\left(I-S \bar{S}^{T}\right)\right]^{\frac{1}{2}} \frac{\operatorname{det}\left(I-Z \bar{S}^{T}\right) \operatorname{tr}\left[\left(I-Z \bar{S}^{T}\right)^{-1} I_{i j} \bar{S}^{T}\right]}{\operatorname{det}\left(I-Z \bar{S}^{T}\right)} .
$$

Then we obtain

$$
\left|\nabla f_{S}(Z)\right|=\left[\operatorname{det}\left(I-S \bar{S}^{T}\right)\right]^{\frac{1}{2}} \frac{\left|\operatorname{det}\left(I-Z \bar{S}^{T}\right)\right|\left\{\sum_{\substack{1 \leq i \leq m \\ 1 \leq j \leq n}}\left|\operatorname{tr}\left[\left(I-Z \bar{S}^{T}\right)^{-1} I_{i j} \bar{S}^{T}\right]\right|^{2}\right\}^{\frac{1}{2}}}{\left|\operatorname{det}\left(I-Z \bar{S}^{T}\right)\right|} .
$$

By Lemmas 6-8 and (17), we obtain

$$
\left\|f_{S}\right\|_{\mathcal{B}^{\frac{1}{2}\left(\Re_{I}\right)}}=\left|f_{S}(0)\right|+\left[\operatorname{det}\left(I-Z \bar{Z}^{T}\right)\right]^{\frac{1}{2}}\left|\nabla f_{S}(Z)\right| \leq C .
$$

From (18), it follows that $f_{S} \in \mathcal{B}^{\frac{1}{2}}\left(\Re_{I}\right)$ and (15) holds.

Next, we prove (ii). Obviously, we have

$$
\frac{\partial g_{S}(Z)}{\partial z_{i j}}=\left[\operatorname{det}\left(I-S \bar{S}^{T}\right)\right]^{\alpha} \frac{\operatorname{det}\left(I-Z \bar{S}^{T}\right) \operatorname{tr}\left[\left(I-Z \bar{S}^{T}\right)^{-1} I_{i j} \bar{S}^{T}\right]}{\left[\operatorname{det}\left(I-Z \bar{S}^{T}\right)\right]^{2 \alpha}} .
$$

Then

$$
\left|\nabla g_{S}(Z)\right|=\left[\operatorname{det}\left(I-S \bar{S}^{T}\right)\right]^{\alpha} \frac{\left|\operatorname{det}\left(I-Z \bar{S}^{T}\right)\right|\left\{\sum_{\substack{1 \leq i \leq m \\ 1 \leq j \leq n}}\left|\operatorname{tr}\left[\left(I-Z \bar{S}^{T}\right)^{-1} I_{i j} \bar{S}^{T}\right]\right|^{2}\right\}^{\frac{1}{2}}}{\left|\operatorname{det}\left(I-Z \bar{S}^{T}\right)\right|^{2 \alpha}} .
$$

Also by Lemmas 6-8 and (19), we have

$$
\left\|g_{S}\right\|_{\mathcal{B}^{\alpha}\left(\Re_{I}\right)}=\left|g_{S}(0)\right|+\left[\operatorname{det}\left(I-Z \bar{Z}^{T}\right)\right]^{\alpha}\left|\nabla g_{S}(Z)\right| \leq C .
$$

From (20), it follows that $g_{S} \in \mathcal{B}^{\alpha}\left(\Re_{I}\right)$ and (16) holds.

Remark 2 It is easy to see that $f_{S}$ and $g_{S}$ uniformly converge to zero on any compact subset of $\Re_{I}$ as $S \rightarrow \partial \Re_{I}$. 


\section{Boundedness and compactness of $M_{\psi}$ on $\mathcal{B}^{\alpha}\left(\Re_{l}\right)$}

First, we have the following result of the operator $M_{\psi}$ on $\mathcal{B}^{\alpha}\left(\Re_{I}\right)$.

Theorem 1 Let $\alpha>0$ and $\psi \in H\left(\Re_{I}\right)$. Then the following statements hold.

(i) For $0<m \alpha<1$, if $\psi \in H^{\infty}\left(\Re_{I}\right) \cap \mathcal{B}^{\alpha}\left(\Re_{I}\right)$, then the operator $M_{\psi}$ is bounded on $\mathcal{B}^{\alpha}\left(\Re_{I}\right)$.

(ii) For $m \alpha=1$, if $\psi \in H^{\infty}\left(\Re_{I}\right)$ and

$$
M_{1}:=\sup _{Z \in \Re_{I}}\left[\operatorname{det}\left(I-Z \bar{Z}^{T}\right)\right]^{\alpha}|\nabla \psi(Z)| \log \frac{2}{\operatorname{det}\left(I-Z \bar{Z}^{T}\right)}<+\infty,
$$

then the operator $M_{\psi}$ is bounded on $\mathcal{B}^{\alpha}\left(\Re_{I}\right)$.

(iii) For $m \alpha>1$, if $\psi \in H^{\infty}\left(\Re_{I}\right)$ and

$$
M_{2}:=\sup _{Z \in \Re_{I}} \frac{|\nabla \psi(Z)|}{\left[\operatorname{det}\left(I-Z \bar{Z}^{T}\right)\right]^{m \alpha-\alpha-1}}<+\infty
$$

then the operator $M_{\psi}$ is bounded on $\mathcal{B}^{\alpha}\left(\Re_{I}\right)$.

(iv) If the operator $M_{\psi}$ is bounded on $\mathcal{B}^{\alpha}\left(\Re_{I}\right)$, then $\psi \in H_{\alpha}^{\infty}\left(\Re_{I}\right) \cap \mathcal{B}^{\alpha}\left(\Re_{I}\right)$.

Proof We first prove (i). For $f \in \mathcal{B}^{\alpha}\left(\Re_{I}\right)$, by Lemma 4, we have

$$
\begin{aligned}
{\left[\operatorname{det}\left(I-Z \bar{Z}^{T}\right)\right]^{\alpha}\left|\nabla\left(M_{\psi} f\right)(Z)\right| } & =\left[\operatorname{det}\left(I-Z \bar{Z}^{T}\right)\right]^{\alpha}|\nabla \psi(Z) f(Z)+\psi(Z) \nabla f(Z)| \\
& \leq\left[\operatorname{det}\left(I-Z \bar{Z}^{T}\right)\right]^{\alpha}(|\nabla \psi(Z) f(Z)|+|\psi(Z) \nabla f(Z)|) \\
& \leq C\|f\|_{\mathcal{B}^{\alpha}\left(\Re_{I}\right)}\|\psi\|_{\mathcal{B}^{\alpha}\left(\Re_{I}\right)}+\|f\|_{\mathcal{B}^{\alpha}\left(\Re_{I}\right)}\|\psi\|_{H^{\infty}\left(\Re_{I}\right)} \\
& =\left(C\|\psi\|_{\mathcal{B}^{\alpha}\left(\Re_{I}\right)}+\|\psi\|_{H^{\infty}\left(\Re_{I}\right)}\right)\|f\|_{\mathcal{B}^{\alpha}\left(\Re_{I}\right)} .
\end{aligned}
$$

Then from (23) we obtain

$$
\begin{aligned}
\left\|M_{\psi} f\right\|_{\mathcal{B}^{\alpha}\left(\Re_{I}\right)} & =|\psi(0) f(0)|+\left[\operatorname{det}\left(I-Z \bar{Z}^{T}\right)\right]^{\alpha}\left|\nabla M_{\psi} f(Z)\right| \\
& \leq\left(|\psi(0)|+C\|\psi\|_{\mathcal{B}^{\alpha}\left(\Re_{I}\right)}+\|\psi\|_{H^{\infty}\left(\Re_{I}\right)}\right)\|f\|_{\mathcal{B}^{\alpha}\left(\Re_{I}\right)} .
\end{aligned}
$$

From the assumption and (24), it follows that the operator $M_{\psi}$ is bounded on $\mathcal{B}^{\alpha}\left(\Re_{I}\right)$.

We prove statement (ii). For each $f \in \mathcal{B}^{\alpha}\left(\Re_{I}\right)$, by Lemmas 2 and 4 , we have

$$
\begin{aligned}
{\left[\operatorname{det}\left(I-Z \bar{Z}^{T}\right)\right]^{\alpha}\left|\nabla\left(M_{\psi} f\right)(Z)\right| } & =\left[\operatorname{det}\left(I-Z \bar{Z}^{T}\right)\right]^{\alpha}|\nabla \psi(Z) f(Z)+\psi(Z) \nabla f(Z)| \\
& \leq\left[\operatorname{det}\left(I-Z \bar{Z}^{T}\right)\right]^{\alpha}(|\nabla \psi(Z)||f(Z)|+|\psi(Z)||\nabla f(Z)|) \\
& \leq\left(M_{1}+\|\psi\|_{H^{\infty}\left(\Re_{I}\right)}\right)\|f\|_{\mathcal{B}^{\alpha}\left(\Re_{I}\right)} .
\end{aligned}
$$

By (25), we obtain

$$
\left\|M_{\psi} f\right\|_{\mathcal{B}^{\alpha}\left(\Re_{I}\right)}=|\psi(0) f(0)|+\sup _{Z \in \Re_{I}}\left[\operatorname{det}\left(I-Z \bar{Z}^{T}\right)\right]^{\alpha}\left|\nabla\left(M_{\psi} f\right)(Z)\right| \leq C\|f\|_{\mathcal{B}^{\alpha}\left(\Re_{I}\right)},
$$

which shows that the operator $M_{\psi}$ is bounded on $\mathcal{B}^{\alpha}\left(\Re_{I}\right)$. 
Statement (iii) can be obtained similarly. Here we omit.

Now, we begin to prove (iv). Choose $f(Z) \equiv 1$ on $\mathfrak{\Re}_{I}$. Then by the boundedness of the operator $M_{\psi}$ on $\mathcal{B}^{\alpha}\left(\Re_{I}\right)$, we have

$$
\left[\operatorname{det}\left(I-Z \bar{Z}^{T}\right)\right]^{\alpha}|\nabla \psi(Z)|=\left[\operatorname{det}\left(I-Z \bar{Z}^{T}\right)\right]^{\alpha}\left|\nabla\left(M_{\psi} f\right)(Z)\right| \leq C\|f\|_{\mathcal{B}^{\alpha}\left(\Re_{I}\right)},
$$

which shows

$$
\sup _{Z \in \Re_{I}}\left[\operatorname{det}\left(I-Z \bar{Z}^{T}\right)\right]^{\alpha}|\nabla \psi(Z)|<+\infty
$$

that is, $\psi \in \mathcal{B}^{\alpha}\left(\Re_{I}\right)$. Again applying the boundedness of the operator $M_{\psi}$ on $\mathcal{B}^{\alpha}\left(\Re_{I}\right)$ to the function $g(Z)=z_{11}$, by Lemma 1 and (27) we obtain

$$
\begin{aligned}
{\left[\operatorname{det}\left(I-Z \bar{Z}^{T}\right)\right]^{\alpha}|\psi(Z)| } & =\left[\operatorname{det}\left(I-Z \bar{Z}^{T}\right)\right]^{\alpha}\left|\nabla \psi(Z) z_{11}+\psi(Z)-\nabla \psi(Z) z_{11}\right| \\
& \leq\left[\operatorname{det}\left(I-Z \bar{Z}^{T}\right)\right]^{\alpha}\left(\left|\nabla \psi(Z) z_{11}+\psi(Z)\right|+\left|\nabla \psi(Z) z_{11}\right|\right) \\
& \leq\left\|M_{\psi} g\right\|_{\mathcal{B}^{\alpha}\left(\Re_{I}\right)}+\|\psi\|_{\mathcal{B}^{\alpha}\left(\Re_{I}\right)} .
\end{aligned}
$$

By (28), we have

$$
\sup _{Z \in \Re_{I}}\left[\operatorname{det}\left(I-Z \bar{Z}^{T}\right)\right]^{\alpha}|\psi(Z)|<+\infty
$$

that is, $\psi \in H_{\alpha}^{\infty}\left(\Re_{I}\right)$. Combining (27) and (29), we obtain $\psi \in H_{\alpha}^{\infty}\left(\Re_{I}\right) \cap \mathcal{B}^{\alpha}\left(\Re_{I}\right)$.

Next, we discuss the compactness of the operator $M_{\psi}$ on $\mathcal{B}^{\alpha}\left(\Re_{I}\right)$.

Theorem 2 Let $\alpha>0$ and $\psi$ be the holomorphic function on $\Re_{I}$. Then the following statements hold.

(i) For $0<m \alpha<1$, if $\psi \in H_{0}^{\infty}\left(\Re_{I}\right) \cap \mathcal{B}_{0}^{\alpha}\left(\Re_{I}\right)$, then the operator $M_{\psi}$ is compact on $\mathcal{B}^{\alpha}\left(\Re_{I}\right)$.

(ii) For $m \alpha=1$, if $\psi \in H_{0}^{\infty}\left(\Re_{I}\right) \cap \mathcal{B}^{\alpha}\left(\Re_{I}\right)$ and

$$
\lim _{Z \rightarrow \partial \Re_{I}}\left[\operatorname{det}\left(I-Z \bar{Z}^{T}\right)\right]^{\alpha}|\nabla \psi(Z)| \log \frac{2}{\operatorname{det}\left(I-Z \bar{Z}^{T}\right)}=0,
$$

then the operator $M_{\psi}$ is compact on $\mathcal{B}^{\alpha}\left(\Re_{I}\right)$.

(iii) For $m \alpha>1$, if $\psi \in H_{0}^{\infty}\left(\Re_{I}\right) \cap \mathcal{B}^{\alpha}\left(\Re_{I}\right)$ and

$$
\lim _{Z \rightarrow \partial \Re_{I}} \frac{|\nabla \psi(Z)|}{\left[\operatorname{det}\left(I-Z \bar{Z}^{T}\right)\right]^{m \alpha-\alpha-1}}=0
$$

then the operator $M_{\psi}$ is compact on $\mathcal{B}^{\alpha}\left(\Re_{I}\right)$.

Proof Here we only prove statement (ii). Statements (i) and (iii) can be similarly proved. By Lemma 5 we only need to prove that, if $\left\{f_{i}\right\}$ is a sequence in $\mathcal{B}^{\alpha}\left(\Re_{I}\right)$ such that $\sup _{i \in \mathbb{N}}\left\|f_{i}\right\|_{\mathcal{B}^{\alpha}\left(\Re_{I}\right)} \leq M$ and $f_{i} \rightarrow 0$ uniformly on any compact subset of $\Re_{I}$ as $i \rightarrow \infty$, then 
$\lim _{i \rightarrow \infty}\left\|M_{\psi} f_{i}\right\|_{\mathcal{B}^{\alpha}\left(\Re_{I}\right)}=0$. We observe that $\psi \in H_{0}^{\infty}\left(\Re_{I}\right)$ and condition (30) imply that, for every $\varepsilon>0$, there exists $\sigma>0$ such that on $K=\left\{Z \in \Re_{I}\right.$ : $\left.\operatorname{dist}\left(Z, \partial \Re_{I}\right)<\sigma\right\}$ it follows that

$$
|\psi(Z)|<\varepsilon \quad \text { and } \quad\left[\operatorname{det}\left(I-Z \bar{Z}^{T}\right)\right]^{\alpha}|\nabla \psi(Z)| \log \frac{2}{\operatorname{det}\left(I-Z \bar{Z}^{T}\right)}<\varepsilon .
$$

For such $\varepsilon$ and $\sigma$, by using (32) and Lemma 4, we have

$$
\begin{aligned}
\left\|M_{\psi} f_{i}\right\|_{\mathcal{B}^{\alpha}\left(\Re_{I}\right)}= & \left|\psi(0) f_{i}(0)\right|+\sup _{Z \in \Re_{I}}\left[\operatorname{det}\left(I-Z \bar{Z}^{T}\right)\right]^{\alpha}\left|\nabla M_{\psi} f_{i}(Z)\right| \\
= & \left|\psi(0) f_{i}(0)\right|+\sup _{Z \in \Re_{I}}\left[\operatorname{det}\left(I-Z \bar{Z}^{T}\right)\right]^{\alpha}\left|\nabla \psi(Z) f_{i}(Z)+\psi(Z) \nabla f_{i}(Z)\right| \\
\leq & \left|\psi(0) f_{i}(0)\right| \\
& +\left(\sup _{Z \in K}+\sup _{Z \in \Re_{I} \backslash K}\right)\left[\operatorname{det}\left(I-Z \bar{Z}^{T}\right)\right]^{\alpha}\left|\nabla \psi(Z) f_{i}(Z)+\psi(Z) \nabla f_{i}(Z)\right| \\
\leq & \left|\psi(0) f_{i}(0)\right|+M \sup _{Z \in K}\left[\operatorname{det}\left(I-Z \bar{Z}^{T}\right)\right]^{\alpha}|\nabla \psi(Z)| \log \frac{2}{\operatorname{det}\left(I-Z \bar{Z}^{T}\right)} \\
+ & \|\psi\|_{\mathcal{B}^{\alpha}\left(\Re_{I}\right)} \sup _{Z \in \Re_{I} \backslash K}\left|f_{i}(Z)\right| \\
& +M \sup _{Z \in K}|\psi(Z)|+\|\psi\|_{H_{\alpha}^{\infty}\left(\Re_{I}\right)} \sup _{Z \in \Re_{i} \backslash K}\left|\nabla f_{i}(Z)\right| .
\end{aligned}
$$

It is obvious to see that if $f_{i} \rightarrow 0$ uniformly on a compact subset of $\Re_{I}$ as $i \rightarrow \infty$, then $\left|\nabla f_{i}(Z)\right|$ does as $i \rightarrow \infty$. Since $\Re_{I} \backslash K$ is a compact subset of $\Re_{I}, f_{i} \rightarrow 0$ uniformly on $\Re_{I} \backslash K$ as $i \rightarrow \infty$. From this and (33), we get

$$
\lim _{i \rightarrow \infty}\left\|M_{\psi} f_{i}\right\|_{\mathcal{B}^{\alpha}\left(\Re_{I}\right)}=0,
$$

which shows that $M_{\psi}$ is compact on $\mathcal{B}^{\alpha}\left(\Re_{I}\right)$.

Theorem 3 Let $\alpha>0$ and $\psi \in H\left(\Re_{I}\right)$. Then the following statements hold.

(i) For $\alpha=\frac{1}{2}$, if the operator $M_{\psi}$ is compact on $\mathcal{B}^{\alpha}\left(\Re_{I}\right)$, then

$$
\begin{aligned}
& \lim _{Z \rightarrow \partial \Re_{I}} \operatorname{det}\left(I-Z \bar{Z}^{T}\right)\left\{\sum_{\substack{1 \leq i \leq m \\
1 \leq j \leq n}} \mid \frac{\partial \psi(Z)}{\partial z_{i j}} \log \frac{2}{\operatorname{det}\left(I-Z \bar{Z}^{T}\right)}\right. \\
& \left.+\left.\psi(Z) \operatorname{tr}\left[\left(I-Z \bar{Z}^{T}\right)^{-1} I_{i j} \bar{Z}^{T}\right]\right|^{2}\right\}^{\frac{1}{2}}=0 .
\end{aligned}
$$

(ii) For $\alpha \neq \frac{1}{2}$, if the operator $M_{\psi}$ is compact on $\mathcal{B}^{\alpha}\left(\Re_{I}\right)$, then

$$
\lim _{Z \rightarrow \partial \Re_{I}} \operatorname{det}\left(I-Z \bar{Z}^{T}\right)\left\{\sum_{\substack{1 \leq i \leq m \\ 1 \leq j \leq n}}\left|\frac{1}{2 \alpha-1} \frac{\partial \psi(Z)}{\partial z_{i j}}+\psi(Z) \operatorname{tr}\left[\left(I-Z \bar{Z}^{T}\right)^{-1} I_{i j} \bar{Z}^{T}\right]\right|^{2}\right\}^{\frac{1}{2}}=0 .
$$

Proof We first prove statement (i). Suppose that the operator $M_{\psi}$ is compact on $\mathcal{B}^{\alpha}\left(\Re_{I}\right)$. Consider a sequence $\left\{S_{n}\right\}$ in $\Re_{I}$ such that $S_{n} \rightarrow \partial \Re_{I}$ as $n \rightarrow \infty$. Using this sequence, we 
define the functions

$$
f_{n}(Z)=\left[\operatorname{det}\left(I-S \bar{S}^{T}\right)\right]^{\frac{1}{2}} \log \frac{2}{\operatorname{det}\left(I-Z{\overline{S_{n}}}^{T}\right)} .
$$

Then, by Lemma 9, we know that the sequence $\left\{f_{n}\right\}$ is uniformly bounded in $\mathcal{B}^{\alpha}\left(\Re_{I}\right)$ and uniformly converges to zero on any compact subset of $\Re_{I}$ as $n \rightarrow \infty$. Hence, by Lemma 5 ,

$$
\lim _{n \rightarrow \infty}\left\|M_{\psi} f_{n}\right\|_{\mathcal{B}^{\alpha}\left(\Re_{I}\right)}=0 .
$$

Using (35), it follows from a direct computation that (i) holds.

\section{Consider}

$$
g_{n}(Z)=\frac{1}{1-2 \alpha} \frac{\left[\operatorname{det}\left(I-S_{n}{\overline{S_{n}}}^{T}\right)\right]^{\alpha}}{\left[\operatorname{det}\left(I-Z{\overline{S_{n}}}^{T}\right)\right]^{2 \alpha-1}} .
$$

We can similarly prove statement (ii) and the details are omitted.

\section{Acknowledgements}

The author would like to thank the anonymous referee for providing valuable comments for the improvement of this paper. This work was supported by the Sichuan Science and Technology Program (2018JY0200) and the Sichuan Province University Key Laboratory of Bridge Non-destruction Detecting and Engineering Computing (2016QZJ01).

\section{Funding}

Not applicable.

\section{Availability of data and materials}

Not applicable.

\section{Competing interests}

The authors declare that they have no competing interests.

\section{Authors' contributions}

All authors contributed equally to the writing of this paper. All authors read and approved the manuscript.

\section{Publisher's Note}

Springer Nature remains neutral with regard to jurisdictional claims in published maps and institutional affiliations.

Received: 19 June 2019 Accepted: 17 July 2020 Published online: 01 August 2020

\section{References}

1. Allen, R.F., Colonna, F.: Weighted composition operators from $H^{\infty}$ to the Bloch space of a bounded homogeneous domain. Integral Equ. Oper. Theory 66, 21-40 (2010)

2. Colonna, F., Li, S.: Weighted composition operators from the minimal Möbius invariant space into the Bloch space. Mediterr. J. Math. 10(1), 395-409 (2013)

3. Cowen, C.C., MacCluer, B.D.: Composition Operators on Spaces of Analytic Functions. CRC Press, Boca Roton (1995)

4. Esmaeili, K., Lindström, M.: Weighted composition operators between Zygmund type spaces and their essential norms. Integral Equ. Oper. Theory 75, 473-490 (2013)

5. Guo, Y.T. Shang, Q.L., Zhang, X.J.: The pointwise multiplier on the normal weight Zygmund space in the unit ball. Acta Math. Sci. 36A(6), 1041-1048 (2018)

6. He, W.X., Li, Y.Z.: Bers-type spaces and composition operators. Northeast. Math. J. 18(3), 223-232 (2002)

7. Hu, P.Y., Shi, J.H.: Multipliers on Dirichlet type spaces. Acta Math. Sin. 17, 263-272 (2001)

8. Kucik, A.S.: Weighted composition operators on spaces of analytic functions on the complex half plane. Complex Anal. Oper. Theory 12, 1817-1833 (2018)

9. Lu, Q.K.: The Classical Manifolds and the Classical Domains. Shanghai Scientific and Technical Publisher, Shanghai (1963) (in Chinese)

10. Stegenga, D.A.: Multipliers of the Dirichlet space. III. J. Math. 24, 113-139 (1980)

11. Stević, S.: Essential norms of weighted composition operators from the $\alpha$-Bloch space to a weighted-type space on the unit ball. Abstr. Appl. Anal. 2008, Article ID 279691 (2008)

12. Stević, S.: Norm of weighted composition operators from Bloch space to $H^{\infty}$ on the unit ball. Ars Comb. 88, 125-127 (2008) 
13. Stević, S.: On a new integral-type operator from the weighted Bergman space to the Bloch-type space on the unit ball. Discrete Dyn. Nat. Soc. 2008, Article ID 154263 (2008)

14. Stević, S.: On a new operator from $H^{\infty}$ to the Bloch-type space on the unit ball. Util. Math. 77, 257-263 (2008)

15. Stević, S.: On a new operator from the logarithmic Bloch space to the Bloch-type space on the unit ball. Appl. Math. Comput. 206, 313-320 (2008)

16. Stević, S.: Norm of weighted composition operators from $\alpha$-Bloch spaces to weighted-type spaces. Appl. Math. Comput. 215, 818-820 (2009)

17. Stević, S.: Weighted composition operators from Bergman-Privalov-type spaces to weighted-type spaces on the unit ball. Appl. Math. Comput. 217, 1939-1943 (2010)

18. Stević, S., Jiang, Zh.J.: Differences of weighted composition operators on the unit polydisk. Mat. Sb. 52(2), 358-371 (2011)

19. Stević, S., Sharma, A.K.: Weighted composition operators between growth spaces of the upper half-plane. Util. Math. 84, 265-271 (2011)

20. Su, J.B., Miao, X., Li, H.J.: Generalization of Hua's inequalities and application. J. Math. Inequal. 9(1), 27-45 (2015)

21. Taylor, G.D.: Multipliers on $D_{\alpha}$. Trans. Am. Math. Soc. 123, 229-240 (1966)

22. Yin, W.P.: The Bergman kernels on super-Cartan domain of the first type. Sci. China Ser. A 43, 13-21 (2000)

23. Zhang, X.J.: The pointwise multipliers of Bloch type space $\beta^{p}$ and Dirichlet type space $D^{q}$ on the unit ball of $\mathbb{C}^{n}$. J. Math. Anal. Appl. 285, 376-386 (2003)

24. Zhou, Z.H., Shi, J.H.: Composition operators on the Bloch spaces in polydisks. Complex Var. Elliptic Equ. 46, 73-88 (2001)

25. Zhou, Z.H., Shi, J.H.: Compactness of composition operators on the Bloch space in the classical bounded symmetric domains. Mich. Math. J. 50, 381-405 (2002)

26. Zhu, K.: Multipliers of BMO in the Bergman metric with applications to Toeplitz operators. J. Funct. Anal. 87, 31-50 (1989)

27. Zhu, K.: Spaces of Holomorphic Functions in the Unit Ball. Springer, New York (2005)

\section{Submit your manuscript to a SpringerOpen ${ }^{\circ}$ journal and benefit from:}

- Convenient online submission

- Rigorous peer review

- Open access: articles freely available online

- High visibility within the field

- Retaining the copyright to your article

Submit your next manuscript at $\gg$ springeropen.com 